\section{Soluble CdS and CdSe Nanorods Synthesized}

Some potential practical applications of semiconductor nanorods-for example, optoelectronic light-emitting diodes for nanowires-require the fabrication of nanorods with precisely specified aspect ratios (length/diameter). However, no method has been reported so far for the synthesis of colloidal semiconductor rods with a narrow distribution of aspect ratios. Researchers at National Taiwan Normal University have synthesized water-soluble crystalline nanorods of CdS and CdSe by using micellar templating in which the shapes of the templates are controlled by the addition of cyclohexane to a micelle suspension.

As reported in the July 2000 issue of Chemistry of Materials, the micelle suspensions were formed by the dissolution of CTAB $\left(\mathrm{CH}_{3}\left(\mathrm{CH}_{2}\right)_{15} \mathrm{~N}\left(\mathrm{CH}_{3}\right)_{3} \mathrm{Br}\right)$ in deionized water. Metal and chalcogenide precursors $\left(\mathrm{CdC}_{12}, \mathrm{Na}_{2} \mathrm{~S}\right.$, or $\left.\mathrm{Na}_{2} \mathrm{Se}\right)$ were then added to form two precursor suspensions. The researchers added various amounts of cyclohexane $(0-0.1 \%$ by volume) to these suspensions, followed by sonication. They slowly added the chalcogenide suspensions to the metal suspensions, and the resulting mixture was sonicated and centrifuged to separate out the suspended product.

Transmission electron micrographs

\section{Review Articles}

The April 21 issue of Science features the following review articles on Correlated Electron Systems: Y. Tokura and N. Nagaosa, "Orbital Physics in Transition-Metal Oxides"; J. Orenstein and A.J. Millis, "Advances in the Physics of High-Temperature Superconductivity"; S. Sachdev, "Quantum Criticality: Competing Ground States in Low Dimensions"; and P.W. Anderson, "Sources of Quantum Protection in High- $\mathrm{T}_{\mathrm{c}}$ Superconductivity."

The July issue of Reviews of Modern Physics contains the following review articles: J.J. Rehr and R.C. Albers, "Theoretical Approaches to X-ray Absorption Fine Structure"; $\mathrm{H}$. Feldmeier and J. Schnack, "Molecular Dynamics for Fermions"; S. Hofmann and G. Münzenberg, "The Discovery of the Heaviest Elements"; J.E. Sonier, J.H. Brewer, and R.F. Kiefl, "muSR Studies of the Vortex State in Type II Superconductors"; J.-L. Viovy, "Electrophoresis of DNA and Other Polyelectrolytes: Physical Mechanisms." showed that the reaction products consist of crystalline nanorods as well as spherical and irregularly shaped nanocrystalline materials. The TEM images revealed CdSe and $\mathrm{CdS}$ samples with the mean aspect ratios of $\sim 6$. A high-resolution TEM image of the CdS nanorods showed an average diameter of $\sim 9 \mathrm{~nm}$. Although further work is needed, the results suggest that rod aspect ratio increases with increasing amounts of added cyclohexane. HRTEM images indicated that CdS and CdSe nanocrystals have wurtzite and zincblende structures, respectively. Selectedarea dispersive x-ray studies of the $\mathrm{CdS}$ nanocrystals showed a Cd:S ratio of 1:1.

The addition of cyclohexane is thought to affect the shapes of the micelles and, therefore, the nanocrystals that form inside them. While the exact role of the hydrocarbon is not known, according to the researchers, one possibility is that it induces a micelle-shape transition. Such morphological changes have been previously observed in CTAB micelle suspensions with other hydrocarbons. Another possibility, according to the researchers, is that bilayer vesicles form in the suspension under intense sonication, and "cyclohexane molecules incorporate into the bilayers and subsequently induce the morphologic changes of the bilayer vesicles."

GREG KHITROV

\section{Magnetoresistance and Differential Conductance in Multiwalled Carbon Nanotubes}

In an attempt to determine whether carbon nanotubes conduct current ballistically or diffusively, researchers from Chonbuk National University, the Korean Research Institute of Standards and Science, and the Electronics and Telecommunication Research Institute have measured magnetoresistance and differential conductance $(\mathrm{dI} / \mathrm{dV})$ as a function of a magnetic field applied perpendicular to the tube axis. As reported in the June 15 issue of Physical Review $B$, these measurements provided evidence that the source of the negative magnetoresistance and differential conduction is the change in the density of states (DOS) near the Fermi level as a function of the applied magnetic field.

Two multiwalled carbon nanotube samples synthesized by an arc-discharge method were prepared on a $\mathrm{SiO}_{2}-\mathrm{Si}$ substrate. The researchers patterned electrical leads using e-beam lithography. They then deposited $20 \mathrm{~nm}$ of $\mathrm{Ti}$ and $50 \mathrm{~nm}$ of $\mathrm{Au}$ onto the contacts by using a thermal evaporation technique. They then annealed the sample systems at temperatures between $600^{\circ} \mathrm{C}$ and $800^{\circ} \mathrm{C}$ for $30 \mathrm{~s}$ to produce ohmic contacts.

Two samples with different voltageprobe separation distances and contact resistances underwent changes in magnetoresistance as a function of the applied field. These changes became more pronounced as the experimental temperature decreased. Experimental temperatures were in the vicinity of $35 \mathrm{~K}$. Two regions of interest exist: the point of maximum magnetoresistance and the point of minimum magnetoresistance. The maximum point was temperature-dependent, that is, magnetoresistance increased as the temperature decreased. Magnetoresistance at the minimum point was temperatureinvariant.

At the maximum point, differential conductance is highly nonlinear. A plot of $\mathrm{dI} / \mathrm{dV}$ versus voltage revealed the depletion of states near the Fermi level. Conductance is therefore concluded to be a function of the DOS near the Fermi level. Magnetoresistance is found to be a function of both applied current and ambient temperature.

At the minimum point (applied field was $7 \mathrm{~T}$ for one sample, $4 \mathrm{~T}$ for the other), $\mathrm{dI} / \mathrm{dV}$ was constant, and therefore the nanotubes exhibited ohmic behavior. Magnetoresistance was no longer a function of applied current. The measured conductance approaches the theoretical value (of $2 G_{0}=4 e^{2} / \mathrm{h}$ ), indicating conduction primarily through only the outer graphene wall and thus is not a function of the electronic DOS.

The researchers concluded that lowtemperature conduction of carbon nanotubes is governed by "magnetic-fieldinduced band transport, rather than the quantum interference effect."

JUNE LAU

\section{Carbon Nanotube Electric Charge Divides into Charge "Islands"}

Physicists Sander Tans and Cees Dekker of the Quantum Transport section at Delft University of Technology have determined that the electrical charge in a semiconductive carbon nanotube is not evenly distributed, but rather is divided into separate charge "islands."

Dekker said, "This insight is important if conductive nanotubes are eventually to be used in electric circuits. We had already made a transistor from a single nanotube and now wanted to know how the charge changes over the length of the tube. Such a charge profile could help us understand how the nanotube transistor works."

As they reported in the April 20 issue of Nature, Tans and Dekker sent an electrical 